\title{
Design of Biomechanical Legs with a Passive Toe Joint for Enhanced Human-like Walking
}

\author{
O. ElDirdiry, R. Zaier* and A. Al-Yahmedi \\ Department of Mechanical and Industrial Engineering, College of Engineering, Sultan Qaboos University, Muscat, Oman.
}

Received 5 March 2017; Accepted 1 October 2017

\begin{abstract}
This paper presents the design procedure of a biomechanical leg, with a passive toe joint, which is capable of mimicking the human walking. This leg has to provide the major features of human gait in the motion trajectories of the hip, knee, ankle, and toe joints. Focus was given to the approach of designing the passive toe joint of the biomechanical leg in its role and effectiveness in performing human like motion. This study was inspired by experimental and theoretical studies in the fields of biomechanics and robotics. Very light materials were mainly used in the design process. Aluminum and carbon fiber parts were selected to design the proposed structure of this biomechanical leg, which is to be manufactured in the Mechanical Lab of the Sultan Qaboos University (SQU). The capabilities of the designed leg to perform the normal human walking are presented. This study provides a noteworthy and unique design for the passive toe joint, represented by a mass-spring damper system, using torsion springs in the foot segment. The working principle and characteristics of the passive toe joint are discussed. Four-designed cases, with different design parameters, for the passives toe joint system are presented to address the significant role that the passive toe joint plays in human-like motion. The dynamic motion that is used to conduct this comparison was the first stage of the stance motion. The advantages of the presence of the passive toe joint in gait, and its effect on reducing the energy consumption by the other actuated joints are presented and a comparison between the four-designed cases is discussed.
\end{abstract}

Keywords: Design procedure; Biomechanical leg; Passive joint; Human-like dynamic; Locomotion; Gait; Toe joint.

$$
\text { تصميم سيقان الية حيوية بأصبع قدم ذو مفصل تفاعلي لتحسـين محاكاة مشي الانسان }
$$

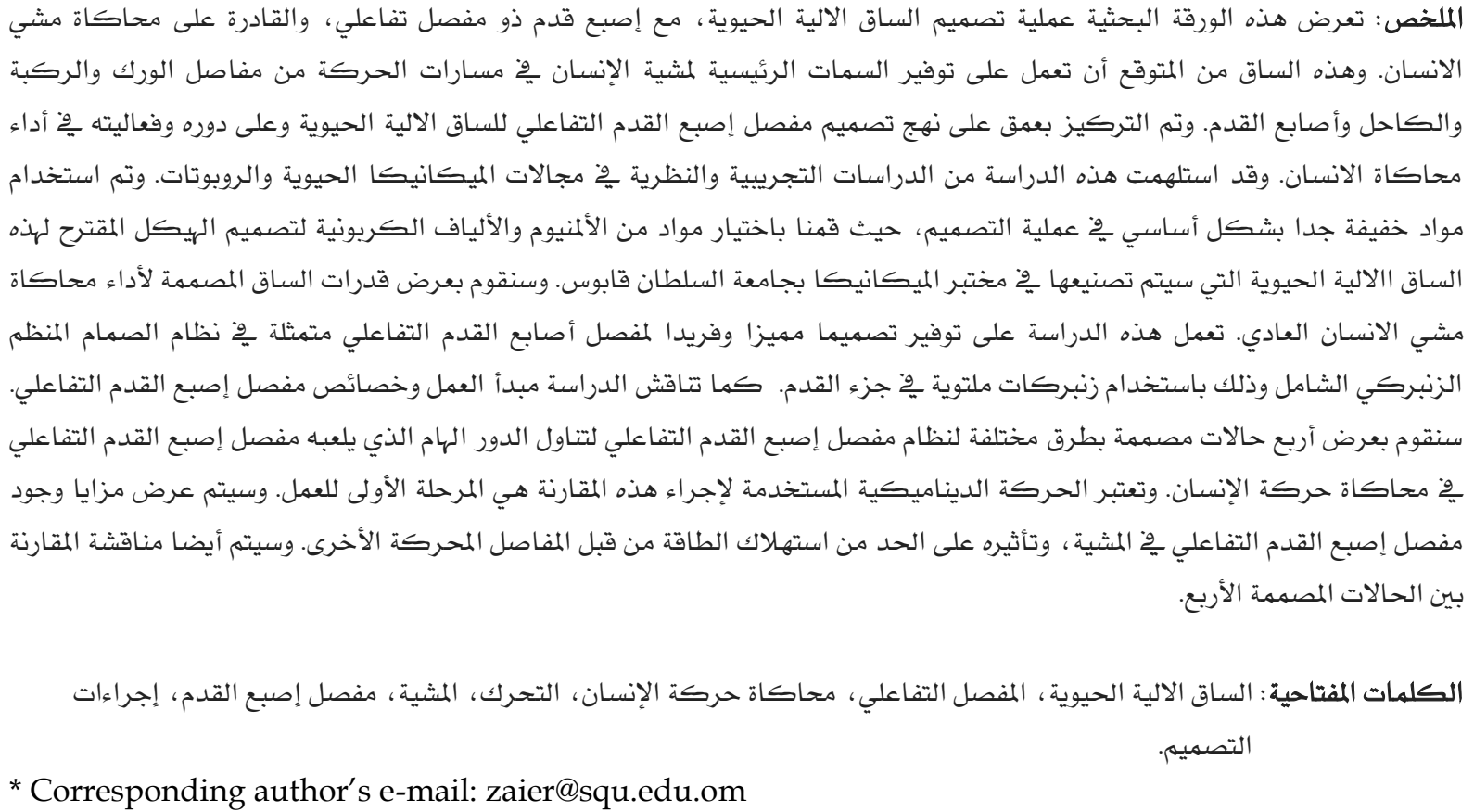

* Corresponding author's e-mail: zaier@squ.edu.om 


\section{Introduction}

During the last few decades, the fields of biomechanics and robotics have witnessed a significant improvement in the designs of the biomechanical legs and prostheses. Moreover, many studies have focused on building humanoid robots in order to mimic human motions (Denny et al. 2016). There are two classes of legged robots; powered and passive legs. The robotic leg to be considered in this study is a powered one. The joints of the robotic legs of this type are usually driven with electric actuators, unlike the passive legs that rely on the force of gravity to perform their stable gait on the ground. It is essential for passive legged robots to have a declining slope (Collins et al. 2001; McGeer 1990). However, if these legs are needed to operate on the ground level, an external force, must be applied to these legs to give them the initial movement (Collins et al. 2005; Wisse and Van Frankenhuyzen 2006). Moreover, in some applications, an actuator is necessary to compensate for the energy loss when the foot touches the ground at the heel. Usually, a pitching actuator in the hip joint is used for this purpose (Alghooneh et al. 2016). There are, generally, three main purposes for designing biomechanical legs. The first objective is to use the biomechanical leg as an orthosis for a human leg. This type of leg is mainly used in rehabilitation, where the orthosis functions as a support for the paralysed leg of a human (Allemand et al. 2009; Yabunaka et al. 2013). Since these types of biomechanical leg are used as a pillar for the leg, they are not designed to look anything but artificial. The second type of biomechanical leg is called a prosthesis, which is a device used to replace a missing part of the human body; as with amputee. Very prominent designs for these types are the design of PANTOE 1 in Peking University in China (Zhu et al. 2010), the design of Massachusetts Institute of Technology (MIT) the Prosthetic Foot (Au et al. 2007) and the design in LaPre et al. (2016). The third type of biomechanical leg is used to study the motion of human gait. Examples of these types are the biped robots studied in (Hwang et al. 2016; Otani et al. 2016; Yi et al. 2016; Iida et al. 2009 and Iida et al. 2007). In this paper, a biped robot with biomechanical legs capable of mimicking the main features of human gait was considered, but, to start with, any suitable biomechanical design, we must take into consideration the importance of ground reaction forces, especially when building a biomechanical leg. Therefore, the structure of the foot, that consists of two segments connected together via a toe joint, needs to be designed so that the leg functions like a human leg.

\subsection{Motivation for Design}

There were many factors that motivated this study. The main one was to address the importance of the toe in human locomotion and to deal with pathological gaits because of the many problems that humans may suffer from such as foot drop. Therefore, the design of a biomechanical leg had to be done in such a way that the mechanical leg possesses the main features of a human leg. It was therefore necessary to investigate the role that the toe joint plays during motion. Moreover, this study highlights the impact of this joint in the dynamic behavior of the biomechanical leg in gait. In order to study these features, it was important to produce a design procedure for the biomechanical leg, in general, and for the passive toe joint in particular. Designing the parameters of the passive toe joint allowed for different study cases to be conducted and allowed for comparison of their dynamic behavior.

Compared to the large number of biomechanical legs designs, as reported above, very few investigations have considered this toe joint in their studies (Sellauoti et al. 2006; Wang et al. 2006; Yamamoto et al. 2007) and the remarkable design of the toe joint in (Piazza et al. 2016). The toe joint itself has been represented as either a powered joint (Ezati et al. 2014; Hernández-Santos et al. 2012; Nishiwaki et al. 2002) or as a passive joint (Sellauoti et al. 2006). In this paper, a study case of a biomechanical leg with a passive toe joint represented by torsion springs is considered. Moreover, the design of this toe joint and the control mechanism of its stiffness are represented so that the behaviour of the foot is relatively close to the behaviour of a human foot.

\subsection{Design Objectives and Constraints}

The objective of this study is to propose the design of a biomechanical leg with the closest essential features needed to generate a gait similar to the human one. Furthermore, this leg will be used to study pathological gaits including, but not limited to, antalgic, ataxic, diplegic, drop foot. This paper, mainly, 
addresses the role of the toe joint in generating gait, and therefore, the following constraints have being identified for the design of the leg and these will be considered in the detailed design:

- Relatively similar dimensions to the human leg.

- Made of very light and stiff materials.

- All the joints are capable of performing the same range of motion as in the human joint.

- The toe joint needs to be passive, with the ability to control its design parameters.

This paper represents the design procedure for the proposed biomechanical leg, and it is organized as follows: in Section 2, the proposed mechanical design of the biomechanical leg is discussed, where geometry, materials, and motor selections are presented. Section 2 also describes the examined range of motion of the biomechanical joints leg design and compares them with the range of motion of the human joints. In Section 3, the capability of the simulated biomechanical leg design to perform normal human walking gait is presented. Section 4 focuses on the suggested design of the passive toe joint and its proposed control mechanism. The kinematics and the dynamics of the proposed biomechanical leg are presented in Section 5 and Section 6, respectively. The results of these analyses are discussed in Section 7. Lastly, a summary is presented in Section 8 .

\section{Mechanical Design}

In this section, the design and the components used to build the biomechanical leg are described (Fig. 1(a)).

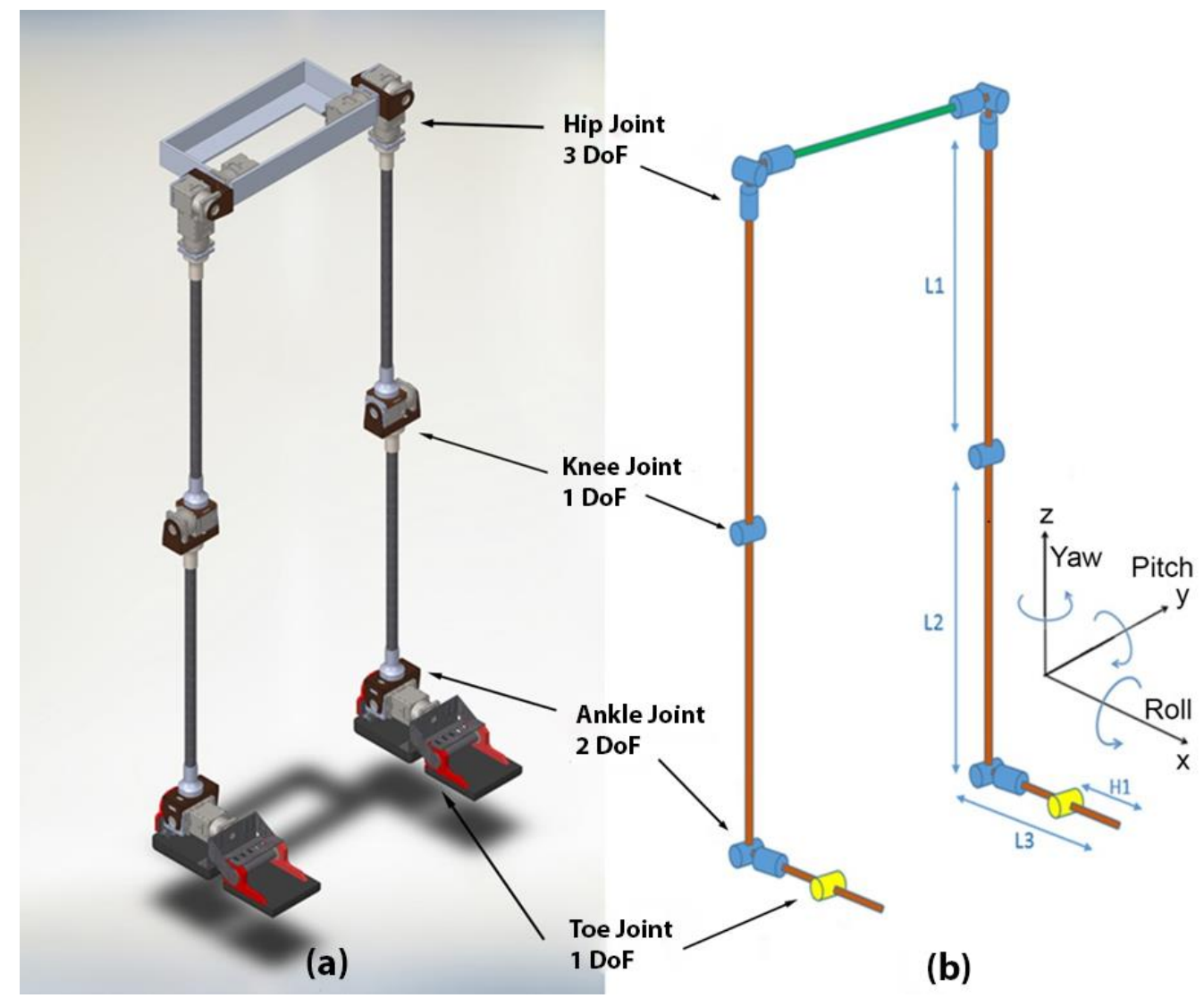

Figure 1. (a) An isometric view for the designed biomechanical legs and (b) Kinematic parameters of the biomechanical legs. 


\subsection{Dimensions, Weights, and Joints Range Motions}

Table 1 and Table 2 compare the average dimensions and weights of human-legs, taken from 100 volunteers in the study (De Leva 1996) with the proposed design of the biomechanical leg. The dimensions were selected, so the design was as close as possible to the human leg, in order to make it easier to mimic the human gait. However, the weight was as light as possible, so that it could be easier to add extra weights if required. For this purpose, aluminum and carbon fiber materials were mainly used to build this design. The dimensions L1, L2, and L3 refer to the lengths of the thigh, the shank, and foot, respectively. To study the foot motion in more detail, the dimensions $\mathrm{H} 1, \mathrm{H} 2$, and $\mathrm{H} 3$ (Fig. 2) refer to some segments in the foot. Moreover, the length of the biomechanical leg had to be similar to the length of a human leg (Table 1). However, the weight of the biomechanical leg needs not be a pivotal issue in order to mimic the human motion, as long as the motors are capable of carrying the body segments and performing the gait.

Table 1. Dimensions comparison in (m).

\begin{tabular}{ccc}
\hline Symbol & $\begin{array}{c}\text { Human Leg (De } \\
\text { Leva 1996) }\end{array}$ & $\begin{array}{c}\text { Biomechanical } \\
\text { Leg }\end{array}$ \\
\hline L1 & 0.41 & 0.41 \\
L2 & 0.44 & 0.44 \\
L3 & 0.26 & 0.26 \\
H1 & 0.09 & 0.09 \\
H2 & 0.05 & 0.04 \\
H3 & 0.07 & 0.06
\end{tabular}

L1, L2, L3 : Thigh, Shank, Foot length

$\mathrm{H} 1=$ Toe length

$\mathrm{H} 2=$ Horizontal distance from back to the ankle of foot.

$\mathrm{H} 3=$ Vertical distance from ankle to the bottom of the foot.

Table 2. Weights comparison in $(\mathrm{kg})$.

\begin{tabular}{ccc}
\hline Segment & $\begin{array}{c}\text { Human } \\
\text { Leg }\end{array}$ & $\begin{array}{c}\text { Biomechanical } \\
\text { Leg }\end{array}$ \\
\hline Thigh & 10 & 0.98 \\
Shank & 3.1 & 0.31 \\
Foot & 1.0 & 1.41 \\
Toe & - & 0.36 \\
\hline
\end{tabular}

De Leva's (1996) study was used to determine the proportionality of heights and the weights for the proposed design segments. The averages for the total body height and weight were selected from De Leva (1996), which are 173.1 $\mathrm{cm}$ and $73 \mathrm{~kg}$, respectively. Hence, the percentage of length and the weight of the leg segments with respect to the total height and weight of the body are found to be as follows:

- Length (thigh $23.2 \%$, shank $24.7 \%$, and foot $4.25 \%)$.

- Weight (thigh $14.16 \%$, shank $4.33 \%$, and foot $1.37 \%)$.

Based on these percentages, the length and the weight of the human leg segments are calculated (Table 1 and Table 2). The actual length and weight for the biomechanical leg segments, after building the design, are given in the same tables. The weights of the thigh and shank of the biomechanical leg are very light compared to the human leg segments. This is due to the very light materials used to build the biochemical leg. However, although very light materials were used to design the biomechanical leg, the foot segment is slightly heavier than the foot segment in the human foot (Table 2), which is due to the large number of parts used to build the foot in the proposed design. Many studies contributed to calculating the average jointsrange motion of human leg e.g. (Grasso et al. 2000). The joint ranges of the human right leg, taken from Grasso et al. (2000), is compared to the joint ranges of the biomechanical leg (Table $3)$. The joint ranges of the biomechanical leg were calculated based on the motion capability of the joints (not causing any collapses) in the CAD design in Solid works. Notice that the joint ranges of a human leg are within the ranges of motion of the biomechanical leg except in the knee (pitching) and the ankle (pitching). All these recorded joint ranges of the biomechanical leg are enough to perform normal human walking.

\subsection{Motors Selection}

Fig. 1(b) describes the kinematic parameters and the motor locations of the biomechanical leg, where three motors ( $3 \mathrm{DOFs}$ ) are used in the hip position, for pitching, rolling and yawing motions. The knee joint is represented by $1 \mathrm{DOF}$ pitching motion, whereas 2 DOF are used in the ankle joint to perform the rolling and 

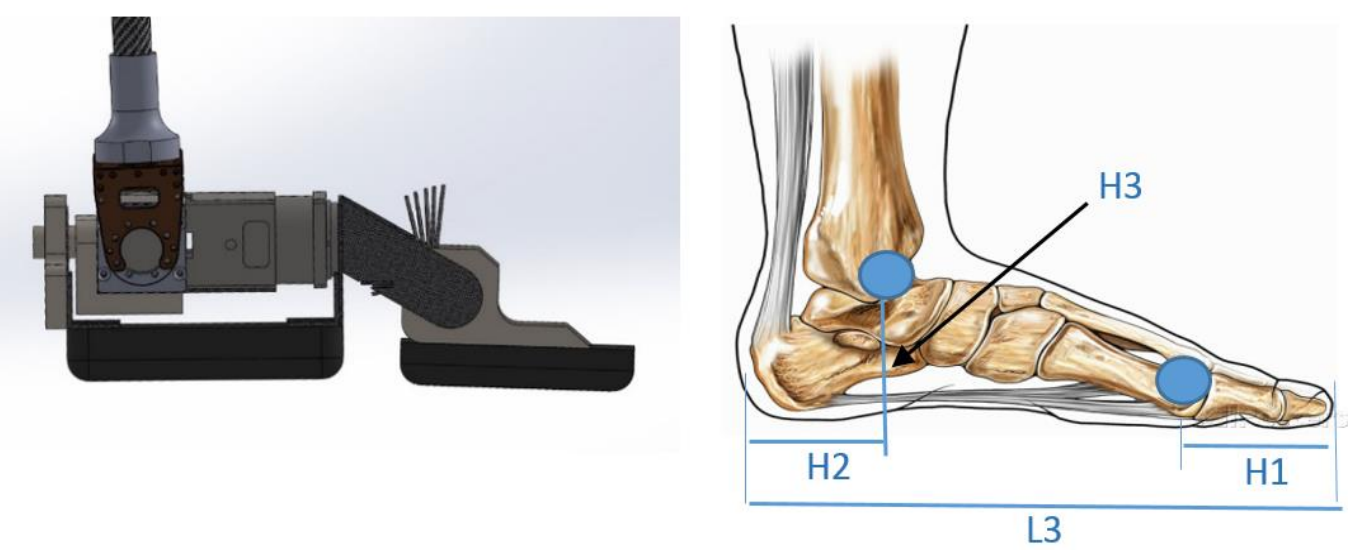

(a)

(b)

Figure 2. Side views for (a) the biomechanical foot and (b) a sketch of human foot.

Table 3. Joints ranges for right leg.

\begin{tabular}{lccc}
\hline & Joint & $\begin{array}{c}\text { Human Leg } \\
\text { (Grasso et al. } \\
2000)\end{array}$ & Biomechanical Leg \\
\hline Hip: & Roll & $-20^{\circ} \mathrm{TO}+40^{\circ}$ & $-25^{\circ} \mathrm{TO}+195^{\circ}$ \\
& Pitch & $-30^{\circ} \mathrm{TO}+110^{\circ}$ & $-180^{\circ} \mathrm{TO}+180^{\circ}$ \\
& Yaw & - & $-180^{\circ} \mathrm{TO}+180^{\circ}$ \\
Knee: & Pitch & $0^{\circ} \mathrm{TO}+150^{\circ}$ & $-105^{\circ} \mathrm{TO}+105^{\circ}$ \\
Ankle: & Roll & $-20^{\circ} \mathrm{TO}+30^{\circ}$ & $-35^{\circ} \mathrm{TO}+35^{\circ}$ \\
& Pitch & $-50^{\circ} \mathrm{TO}+20^{\circ}$ & $-45^{\circ} \mathrm{TO}+25^{\circ}$ \\
Toe (Passive): Pitch & - & $0^{\circ} \mathrm{TO}+70^{\circ}$ \\
\hline
\end{tabular}

Table 4. Maximum toque for the bio-mechanical leg joints.

\begin{tabular}{lcc}
\hline & Joint & $\begin{array}{c}\text { Torque Value } \\
(\mathbf{N m})\end{array}$ \\
\hline Hip: & Roll & 3.87 \\
& Pitch & 5.23 \\
& Yaw & 1.50 \\
Knee: & Pitch & 6.35 \\
Ankle: & Roll & 0.26 \\
& Pitch & 4.48 \\
Toe (Passive): Pitch & 0.61 \\
\hline
\end{tabular}

pitching motions. The toe joint (the only passive joint in this structure) is represented by $1 \mathrm{DOF}$ without any actuator. Table 4 shows the maximum joint torque values that are required to accomplish normal human gait.

DYNAMIXEL PRO motors, provided by Robotis (2017) are used since they can provide the torque values sufficient to perform the gait. It is clear that the pitching movement required more torque than the rolling and yawing movements (Table 4). The motor L42-10-S300-R (DYNAMIXEL PRO motor) was selected to represent the low torque joints, whereas the motor L54-30-S400-R was selected to fulfill the high toque joint (the pitching hip joint and the pitching knee joint required maximum torque of $5.23 \mathrm{Nm}$ and $6.35 \mathrm{Nm}$, respectively). The specifications for these two motors are shown in Fig. 3. Another major factor considered in the selection of these motors is the angular speed of 
motors required to produce a gait similar to human gait. Since the angular velocity for a human joint is, approximately, $20 \mathrm{rpm}$ (Riemer and Shapiro 2011), which is equal to 120 degree per second, these two motors were selected so that they could perform well at this speed.

\section{Biomechanical Behavior and Human Walking Gaits}

In order to mimic human motion using the biomechanical leg, it is important to study the cyclic pattern of human motion. The walking step consists of two phases: the stance phase, which represent about $60 \%$ of the duration of the whole step, and the swing phase, which takes approximately the remaining $40 \%$ (Zhu et al. 2010).

Figure 4 highlights the phases of one step, presented by the proposed biomechanical leg. As shown in the figure, at the beginning of the step, the heel of the biomechanical foot strikes the ground. The bottom part of the leg was made of a rubber material in order to absorb the shock when the heel hits the ground. Then, the pitching motor, at the ankle joint, rotates, causing a flat foot orientation, on the ground level. After that, with the help of the upper structure of the biomechanical leg, the ankle joint pitches in the opposite direction to reach the mid-stance phase of the foot and continues its rotation to reach the heel-off phase. Because of this motion, the heel of the foot will start rising from the ground level, while the toe remains level on the ground. As a result, the passive toe joint stores energy, in a spring element, which is released at the end of the stance phase and, therefore, contributes in lifting the leg. During the swing motion, the ankle pitching actuator returns to its original orientation so that the foot becomes ready for a new stance phase. It is worth mentioning that during this cyclic motion, the upper motors of the hip and the knee joints contribute in maintaining the right orientation to perform the stance and the swing motions.

\section{The Passive Toe Joint}

To address the importance of the presence of the passive toe joint in the foot segment in the biomechanical leg and to enhance locomotion stability, the effectiveness of the passive toe joint during the stance phase should be investigated. The proposed model of the passive toe joint consisting of damper-spring-mass will be presented first. Then, by investigating four values of the stiffness and damping coefficients, a comparison will be made in terms of the amount of energy consumed by the actuators of the ankle and knee joints.

\subsection{Mechanical Structure of the Passive Toe Joint}

In order to reach the design objectives and to achieve the controllability of the passive toe joint parameters, the design in Fig. 5(a) and (b) was selected. In this design, the passive joint consists of five torsion springs, rotating freely around a carbon fiber tube. There are two bearings that support the carbon fiber tube from both sides and allow the pitching rotation in the toe joint. The torque of each spring can be calculated using the following equation:

$\tau=-k_{\mathrm{r}} \theta$

where $\tau$ is the torque applied on the spring in $(\mathrm{Nm})$, and $\theta$ is the twist angle from its original position in radians. $\mathrm{k}_{\mathrm{r}}$ is the rotational stiffness constant (which is also known as spring's torsion coefficient or, torsion elastic modulus) with units of newton-meters/radian. In equation (1), the negative sign represents the direction of the torque with respect to the twist direction. When five springs work in parallel, their rotational stiffness will sum up and give the following rotational spring constant:

$k_{r e q}=k_{\mathrm{r} 1}+k_{\mathrm{r} 2}+k_{\mathrm{r} 3}+k_{\mathrm{r} 4}+k_{\mathrm{r} 5}$

In the structure shown above, there is a carbon fiber sheet with five rooms, one room in front of each spring. This sheet works as a stopper to prevent the upper part (Side A) of the spring from rotating. All the lower side (Side B) of the torsion springs are fixed in the foot. During the gait, when the heel of the biomechanical leg starts to rise and just before the toe leaves the ground (Fig. 4), the lower part of the five springs will start to rotate around the carbon fiber tube. Meanwhile, the upper part of the five springs will enter the rooms of the stopper. This stopper was designed so that two options of rotational stiffness could be provided. The first option is to have a constant 


\begin{tabular}{|c|c|c|c|}
\hline \multicolumn{4}{|l|}{ Data } \\
\hline & & Unit & Value \\
\hline \multicolumn{2}{|l|}{ Dimension } & $\begin{array}{l}\mathrm{mm} \\
\text { (in) }\end{array}$ & $\begin{array}{c}42 \times 42 \times 72 \\
(1.65 \times 1.65 \times 2.83)\end{array}$ \\
\hline \multicolumn{2}{|l|}{ Weight } & $\mathrm{Kg}(\mathrm{oz})$ & $0.269(9.5)$ \\
\hline \multicolumn{2}{|c|}{ Nominal Voltage } & $\mathbf{v}$ & 24 \\
\hline \multicolumn{2}{|c|}{ No Load Speed } & RPM & 28 \\
\hline \multicolumn{2}{|c|}{ No Load Current } & A & 0.52 \\
\hline \multirow{3}{*}{$\begin{array}{l}\text { Continuous } \\
\text { Operation }\end{array}$} & Speed & RPM & 26.0 \\
\hline & Torque & $\begin{array}{l}\text { N.m(oz.f- } \\
\text { in) }\end{array}$ & $1.7(241)$ \\
\hline & Current & $\mathbf{A}$ & 0.6 \\
\hline \multicolumn{2}{|l|}{ Resolution } & Steps/turn & 263168 \\
\hline \multicolumn{2}{|l|}{ Gear Ratio } & - & $257: 1$ \\
\hline \multicolumn{2}{|l|}{ Backlash } & $\operatorname{arcmin}$ & 4.2 \\
\hline \multicolumn{2}{|c|}{ Network Interface } & - & RS-485 \\
\hline \multicolumn{2}{|c|}{$\begin{array}{l}\text { Operating } \\
\text { Temperature }\end{array}$} & ${ }^{\circ} \mathrm{C}$ & $5 \sim 55$ \\
\hline
\end{tabular}

\section{Performance Graph}

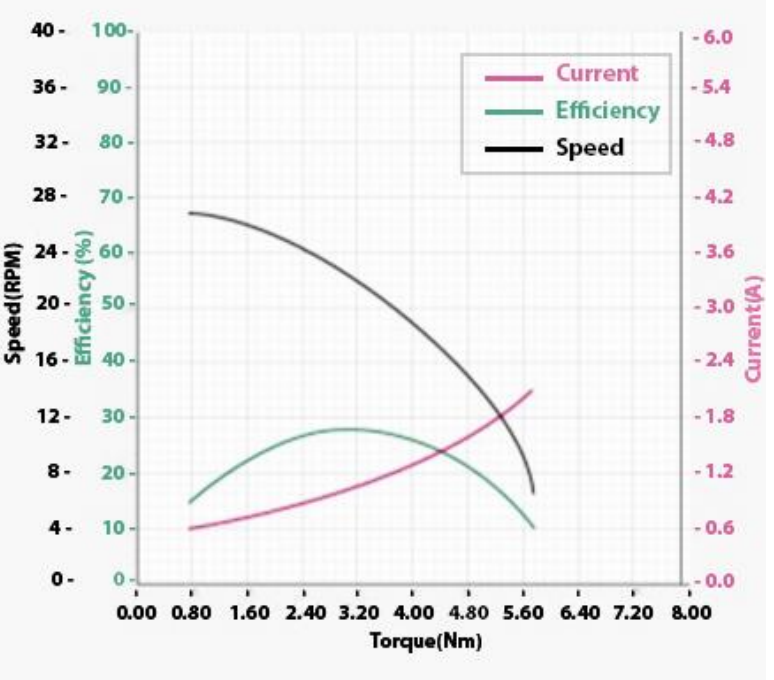

Data

\begin{tabular}{|c|c|c|c|}
\hline & Unit & Value \\
\hline \multicolumn{2}{|l|}{ Dimension } & $\begin{array}{l}\mathrm{mm} \\
\text { (in) }\end{array}$ & $\begin{array}{c}54 \times 54 \times 108 \\
(2.13 \times 2.13 \times 4.25)\end{array}$ \\
\hline \multicolumn{2}{|l|}{ Weight } & $\mathrm{Kg}(\mathrm{Oz})$ & $0.612(21.6)$ \\
\hline \multicolumn{2}{|c|}{ Nominal Voltage } & V & 24 \\
\hline \multicolumn{2}{|c|}{ No Load Speed } & RPM & 28.7 \\
\hline \multicolumn{2}{|c|}{ No Load Current } & A & 1.19 \\
\hline \multirow{3}{*}{$\begin{array}{l}\text { Continuous } \\
\text { Operation }\end{array}$} & Speed & RPM & 26.9 \\
\hline & Torque & $\begin{array}{l}\text { N.m(oz.f- } \\
\text { in) }\end{array}$ & $2.5(354)$ \\
\hline & Current & A & 1.6 \\
\hline \multicolumn{2}{|l|}{ Resolution } & Steps/turn & 288360 \\
\hline \multicolumn{2}{|l|}{ Gear Ratio } & - & 401:1 \\
\hline \multicolumn{2}{|l|}{ Backlash } & arcmin & 4.6 \\
\hline \multicolumn{2}{|c|}{ Network Interface } & - & RS-485 \\
\hline \multicolumn{2}{|c|}{$\begin{array}{l}\text { Operating } \\
\text { Temperature }\end{array}$} & ${ }^{0} \mathrm{C}$ & 5 55 \\
\hline
\end{tabular}

(a)

\section{Performance Graph}

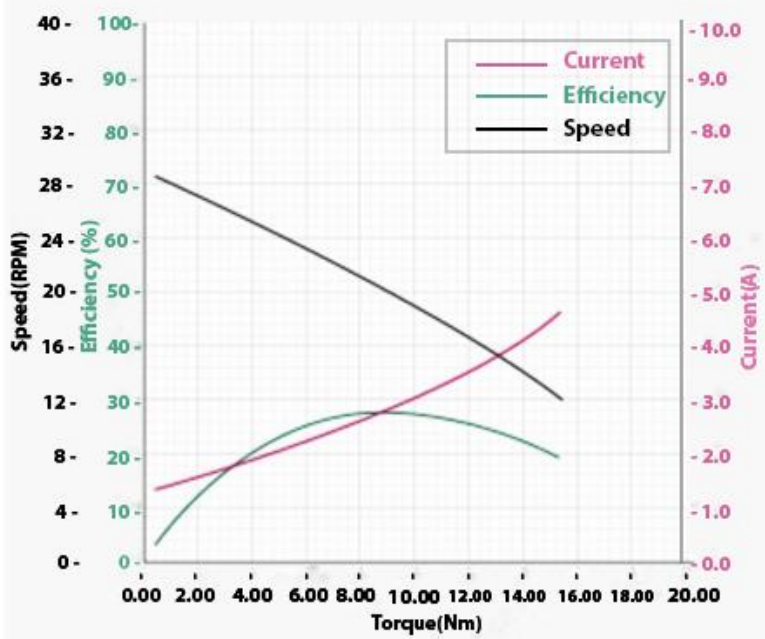

(b)

Figure 3. Snecifications for the DYNAMIXEL PRO motors (a) L42-10-S300-R and (b) L54-30-S400-R

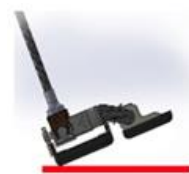

(1)

Heel-Strike

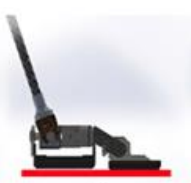

(2)

Foot-Flat

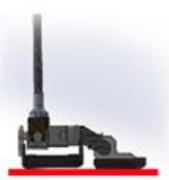

(3)

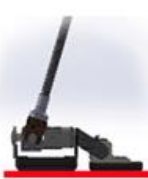

(4)

Heel-off

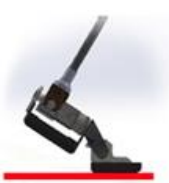

(5)

Toe-off

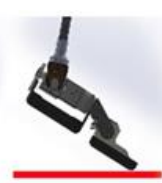

(6)

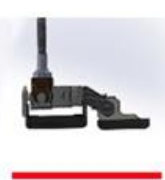

(7)
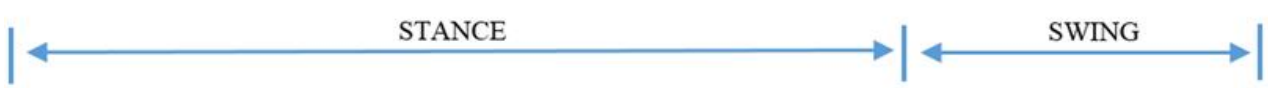

Figure 4. A Simulation of one biomechanical leg during the stance and swing phases of a normal gait. 


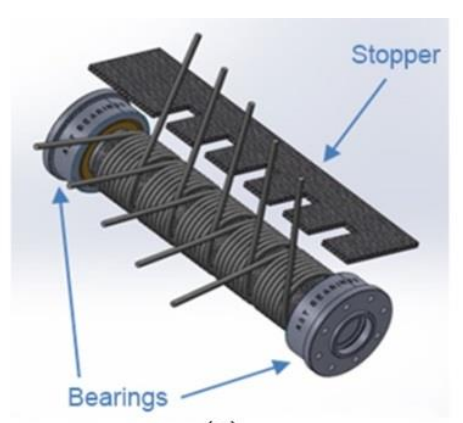

(a)

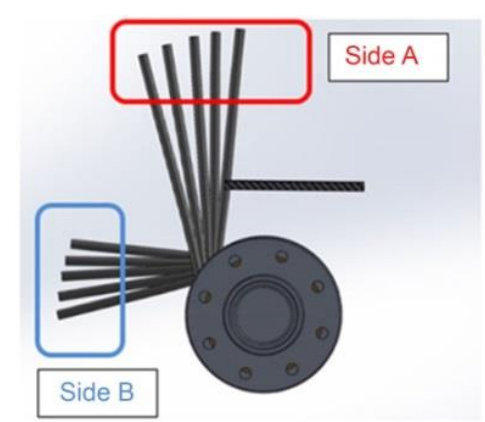

(b)

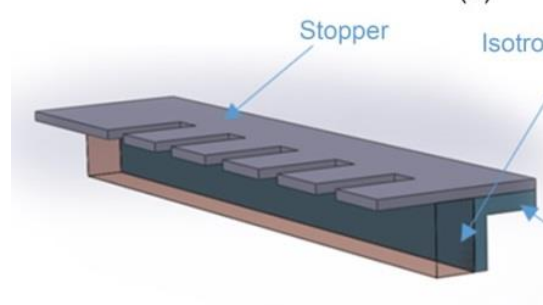

(c)

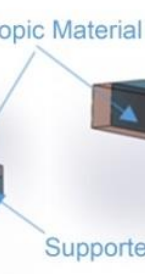

Supporter

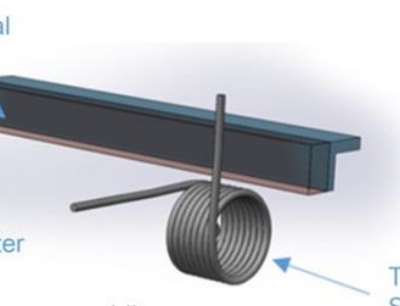

(d)

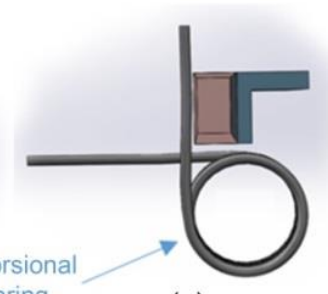

(e)

Figure 5. (a) An isometric material and (b) a side views for the structure of the passive toe joint part for the biomechanical leg. (c) A supporter for the isotropic material is attached below the stopper, (d) the stopped is hidden and one of the five springs is shown in front of the isotropic material, and (e) a side view for the structure.

stiffness when the five torsion springs engage with the carbon fiber sheet rooms simultaneously. The other option is to have a variable stiffness, when the springs are engaged inside the rooms at different times during the gait. It is worth mentioning that the springs' housings for the first option are the same lengths while in the second option they have different lengths (Fig. 5). Also, the carbon fiber sheet (i.e. the stopper) can be easily replaced with another sheet in order to switch between the two rotational stiffness. In this study, a constant value of the rotational spring constant will be considered. The energy $U$ is the stored energy in the spring in joules and it can be calculated using the following equation:

$U=\frac{1}{2} k_{\mathrm{req}} \theta^{2}$

In this design (Fig. 5(c), (d), and (e)) an elastic isotropic material is placed below the stopper and in front of the springs in order to add a damping effect to the system. The springs will be in contact with the isometric material before they reach the stopper. This material can be selected later based on the rotational damping coefficient $c_{r}$, which will also, be calculated later in this study.

\subsection{Passive Toe Joint Model}

During the last stage of the stance phase (Fig. 4) described by the motions between the positions " 4 " and " 5 ", the location of the toe joint is fixed. The role of the springs comes during this period. The proposed design model for the toe joint is represented by a springdamper system (Fig. 6(a)). The mass (M) represents the mass of the whole leg. The line $(r)$ links the toe joint to the heel point, and $\theta$ depicts the angle between the line $(r)$ and the ground level. The motion of the heel point is represented by the arc length $(s)$.

When the biomechanical leg performs the motion, from position " 4 " to position " 5 " (Fig. $4)$, the value of $\theta$ increases. Hence, energy will be stored in the compressed torsion springs in the toe joint. This stored energy will be released allowing the foot to contribute to generating the gait from positions " 5 " and "6" (Fig. 4). In order to manifest the roles of the toe joint, the model was described by the following ordinary differential equation:

$J \ddot{\theta}+c_{\mathrm{r}} \dot{\theta}+k_{\text {req }} \theta=0$,

where $J$ is the polar moment of inertia of the toe joint about the y-axis, $c_{\mathrm{r}}$ is the rotational damping coefficient, $k_{r e q}$ is the rotational spring constant for the five torsion springs. $\theta, \dot{\theta}$ and 
$\ddot{\theta}$ are the angle, the angular velocity, and the angular acceleration of the toe joint, respectively.

By neglecting the mass of the links, the mass that is being conceded in this study is the mass of the torso and the total mass of the other swinging leg, which is $M=5 \mathrm{Kg}$. Therefore, the curved rotational model (Fig. 6(a)) is demonstrated as an equivalence model for the linear model (Fig. 6(b)).

Since the mass $M$ is moving vertically without rotating, the equation of motion will depend only on the displacement y. Hence, the rotational spring $k_{\text {req }}$ needs to be converted to linear one $k_{\text {leq. }}$. The following relation can be used:

$k_{\text {leq }}=k_{\text {req }}\left(\frac{1}{r}\right)^{2}$

Similarly, the relation between the linear damping coefficient $\left(c_{1}\right)$ and the rotational damping coefficient $\left(c_{\mathrm{r}}\right)$ can be calculated, as follows:

$c_{1}=c_{\mathrm{r}}\left(\frac{1}{r}\right)^{2}$

The equation of motion of the mass $M$ can be illustrated in equation (6) in term of the linear elements:

$M \ddot{y}+c_{1} \dot{y}+k_{\text {leq }} y=0$

where $M$ is the mass of the biomechanical leg above the foot segment, including all the mass shown by the Area A. $y, \dot{y}$ and $\ddot{y}$ are the position, the velocity, and the acceleration of the heel point along the $y$-axis with respect to the ground, respectively. An important point to mention is that the spring constant $k_{1}$ and the damping coefficient $c_{1}$ are linear in this case and they are measured in $\mathrm{N} / \mathrm{m}$ and the $\mathrm{Ns} / \mathrm{m}$, respectively. Unlike equation (4), the spring constant and the damping coefficient are measured in $\mathrm{Nm} / \mathrm{rad}$ and $\mathrm{mNs} / \mathrm{rad}$, respectively.

\subsection{System Design for the Passive Toe Joint}

In order to design the toe joint, the desired angle $\theta$ that the toe joint can reach is set. Assuming that, during the last stage of the stance motion from position " 4 " to position " 5 "
(Fig. 4) the toe joint rotates in the clockwise direction from $0^{0}$ to $30^{\circ}$. If the value of $r=0.13 \mathrm{~m}$ then using the relation $y=r \cdot \sin \theta$, the desired change in $y$ along the y-axis will vary from $0 \mathrm{~m}$ to $0.065 \mathrm{~m}$. The solution of Equation (7) is given as follows:

$y(t)=e^{-\xi \omega t}\left(b_{1} e^{j \sqrt{1-\xi^{2}} \omega t}+b_{2} e^{-j \sqrt{1-\xi^{2}} \omega t}\right)$,

where, $b_{1}$ and $b_{2}$ are arbitrary complex-valued constants of integration that can be found by the initial conditions, $\xi$ is non-dimensional number called damping ratio and $\omega$ is the undamped natural frequency in rad per second. The damping coefficient $c_{1}$ and the spring constant $k_{1}$ can be found from the following relations:

$c_{c r}=2 \sqrt{k_{1} M}$,

$c_{c r}=2 M \omega$,

$\xi=\frac{c_{1}}{c_{c r}}$

where $c_{c r}$ is the critical damping value. Therefore, the values of the damping coefficient $c_{1}$ and the spring constant $k_{1}$ can be calculated with the following equations, for given values of $\omega$ and $\xi$ :

$k_{1}=m \omega^{2}$

$c_{1}=2 \xi \sqrt{k M}$

The effect of the value of $\xi$ on the response of the second order system is demonstrated in Inman (2013), where the system is called under damped system when $0<\xi<1$, critically damped system when $\xi=1$, and overdamped system when $1<\xi<2$.

Three values, of $\omega$ and $\xi$, were selected in order to investigate the trajectories of the heel point during 1.5 seconds in the second order system (Fig. 7 and Table 5). For the selected parameters $\omega$ and $\xi$, the values of $c$ and $k$ were calculated, using the equations (12) and (13), and recorded (Table 6).

To study the effect of the passive toe joint in the powered ankle and knee joints, a fourth case for the flat foot without a spring and damper elements (fixed toe joint) was introduced. Then, both the kinematics and dynamics analysis of the biomechanical leg must be studied. 


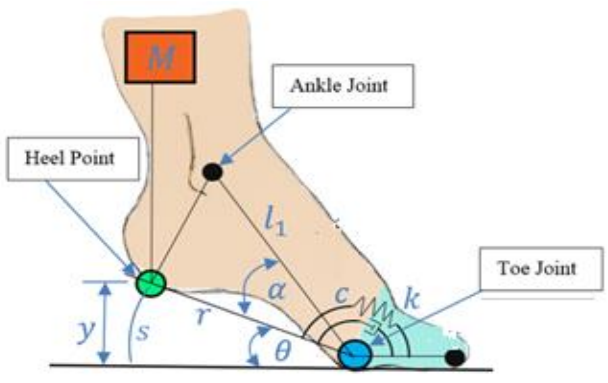

(a)

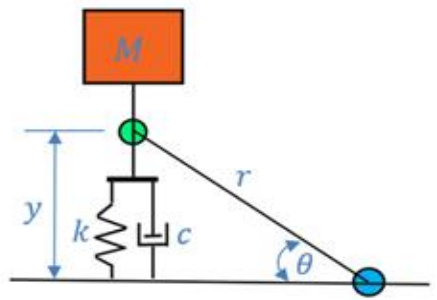

(b)

Figure 6. (a) A sketch of a human foot with mass-spring damper system model, for the toe joint, which uses torsion spring and damper. (b) Equivalent mass-spring damper system model that uses linear spring and damper.

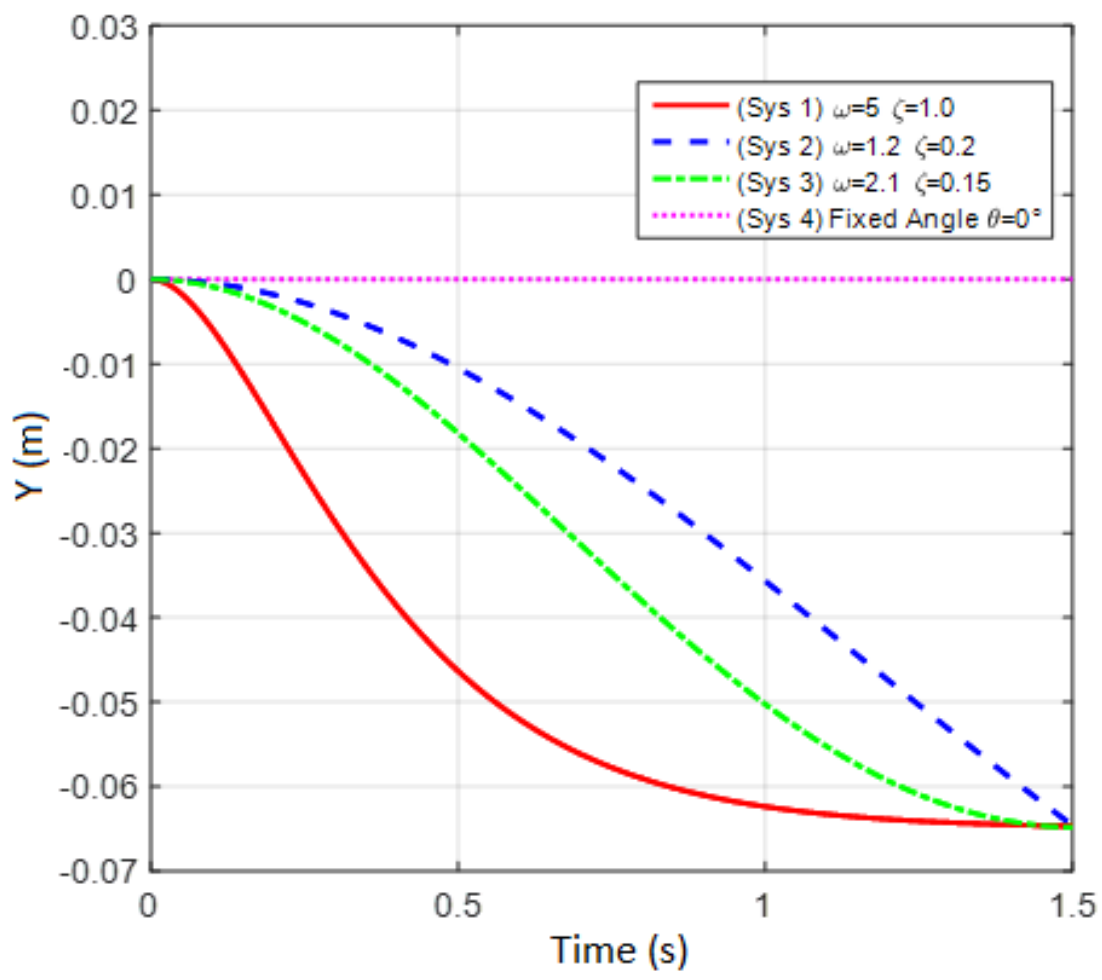

Figure 7. The vertical displacements of the heel point, in the biomechanical foot, caused by the rotational movement of the toe joint by $30^{\circ}$ for case 1 , case 2 , case 3 , and case 4 .

\section{Kinematics Analysis for the Biomechanical Leg.}

Before studying the dynamics and addressing the effect of the passive toe joint on the ankle and knee joints, it is important to solve the inverse kinematic for the whole leg. Since the case when the biomechanical leg is at the end of the stance phase is considered, the location of the toe joint will be taken as the base point (Fig. 8). The torso part will represent the end-effector of the mechanism as the point $\left(X_{T}, Y_{T}\right)$, and it will move based on a predefined motion profile. Since the structure of the proposed biomechanical leg is identical to the structure of the biped robot in Wang et al. (2006), similar equations and methodology will be developed

Table 5. Three cases of damper and springs for toe joint.

\begin{tabular}{cccc}
\hline $\begin{array}{c}\text { Symbol } \\
\text { (Unit) }\end{array}$ & Case $\mathbf{1}$ & Case $\mathbf{2}$ & Case 3 \\
\hline$\xi$ & 1.0 & 0.2 & 0.15 \\
$\omega(\mathrm{Hz})$ & 5.0 & 1.2 & 2.1 \\
\hline
\end{tabular}


in deriving the dynamics and the kinematics of the leg. In this research, the following two relations represent the desired planar motion of the torso:

$X_{T}=a t-0.1$

$Y_{T}=A \sin \left(\frac{\pi t}{2}\right)+L_{2}+L_{3}-L_{4}$

where $a$ is the forward distance for the torso and $A$ is the swing height of the torso motion. In order to achieve a reachable position by the torso during the stance phase, $a$ and $A$ are set to be $0.3 \mathrm{~m}$ and $0.1 \mathrm{~m}$, respectively. $t$ is the time, $L_{2}, L_{3}$, and $L_{4}$ are the lengths of the links in the mechanism (Fig. 8). The angle $\alpha$, which is shown in the same figure, is constant in the design and approximately equal to $\frac{\pi}{9}$.

The toe joint is considered to be fixed in the ground and represented with the angle $\theta_{1}$. Since the position of the heel point " $y$ " was determined in the previous section for different toe joint cases, the path trajectory of $\theta_{1}$ can be found using the relation $\theta=\sin ^{-1}\left(\frac{y}{r}\right)$. Therefore, for a given torso motion, the other pitching angles of ankle, knee and hip joints of the supporting leg can be found as following:

$\theta_{2}=\gamma-\beta-\frac{\pi}{2}+\theta_{1}$

$\theta_{3}=\arccos \left(\frac{\left(X_{T}-L_{1} C_{1}\right)^{2}+\left(Y_{T}-L_{1} S_{1}\right)^{2}-L_{2}^{2}-L_{3}^{2}}{2 L_{2} L_{3}}\right)$

$\theta_{4}=\frac{\pi}{2}-\gamma+\beta-\theta_{1}-\theta_{3}$

From the geometry of the leg, (Fig. 8) $\gamma$ and $\beta$ can be represented by the following equations:

$\gamma=\arctan \left(Y_{T}-L_{1} S_{1}, X_{T}-L_{1} C_{1}\right)$

$\beta=\arccos \left(\frac{\left(X_{T}-L_{1} C_{1}\right)^{2}+\left(Y_{T}-L_{1} S_{1}\right)^{2}+L_{2}^{2}-L_{3}^{2}}{2 L_{2} \sqrt{\left(X_{T}-L_{1} C_{1}\right)^{2}+\left(Y_{T}-L_{1} S_{1}\right)^{2}}}\right)$

where $S_{n}$ and $C_{n}$ in the equations are short forms of $\operatorname{Sin}\left(\theta_{n}\right)$ and $\cos \left(\theta_{n}\right)$, respectively.

\section{Kinematics Analysis for the Biomechanical Leg.}

Before calculating the torque and the output energy of the joints, it is important to address the potential energy $(U)$ and the kinetic energy
$(K)$ of the system. The following equations were used to describe the planar location of the torso $\left(X_{T}, Y_{T}\right)$ :

$X_{T}=L_{1} C_{1}+L_{2} C_{2}+L_{3} C_{3}$

$Y_{T}=L_{1} S_{1}+L_{2} S_{2}+L_{3} S_{3}$

Therefore, the velocity of the torso can be written as:

$\dot{X}_{T}=-L_{1} S_{1} \dot{\theta_{1}}-L_{2} S_{2} \dot{\theta_{2}}-L_{3} S_{3} \dot{\theta_{3}}$

$\dot{Y}_{T}=L_{1} C_{1} \dot{\theta_{1}}+L_{2} C_{2} \dot{\theta}_{2}+L_{3} C_{3} \dot{\theta_{3}}$

where $\dot{\theta_{n}}$ is the angular velocity of the joints. Based on the equations of the position and velocity of the torso and considering the gravity as $g=-9.8 \mathrm{~m} / \mathrm{s}^{2}$, the potential and the kinetic energies of the system can be calculated as follows:

$U=M g Y_{T}=-M g \sum_{n=1}^{3} L_{n} S_{n}$

$K=\frac{1}{2} M\left(\left(\dot{X}_{T}\right)^{2}+\left(\dot{Y}_{T}\right)^{2}\right)$

Then, using Lagrange's dynamical equation:

$\frac{d}{d t}\left(\frac{\partial K}{\partial \dot{\theta_{n}}}\right)-\frac{\partial K}{\partial \theta_{n}}+\frac{\partial U}{\partial \theta_{n}}=T_{n}$

the torque of the system is represented by the following dynamic equation:

$\tau=A \ddot{\theta}+B \dot{\theta^{2}}+G$

where $\tau=\left[\begin{array}{lll}\tau_{1} & \tau_{2} & \tau_{3}\end{array}\right]$ represents the torque of the first three joints, as $\tau_{4}=0$ in the proposed case. The angular acceleration in the equation is $\ddot{\theta}=\left[\begin{array}{lll}\ddot{\theta_{1}} & \ddot{\theta_{2}} & \ddot{\theta_{3}}\end{array}\right]^{T}$, and the square of the angular velocity is $\dot{\theta}^{2}=$ $\left[\begin{array}{ccc}\dot{\theta_{1}} & \dot{\theta_{2}} & \dot{\theta}_{3}^{2}\end{array}\right]^{T}$. The matrices $A, B$ and $G$ of the dynamic equation (28) are calculated as:

Table 6. Calculated damper and springs coefficients for toe joint.

\begin{tabular}{cccc}
\hline $\begin{array}{c}\text { Symbol } \\
\text { (Unit) }\end{array}$ & Case 1 & Case $\mathbf{2}$ & Case 3 \\
\hline$c_{\mathrm{L}}(\mathrm{N} . \mathrm{S} / \mathrm{M})$ & 50 & 2.4 & 3.15 \\
$k_{\mathrm{L}}(\mathrm{N} / \mathrm{M})$ & 125 & 7.2 & 22.05 \\
\hline
\end{tabular}




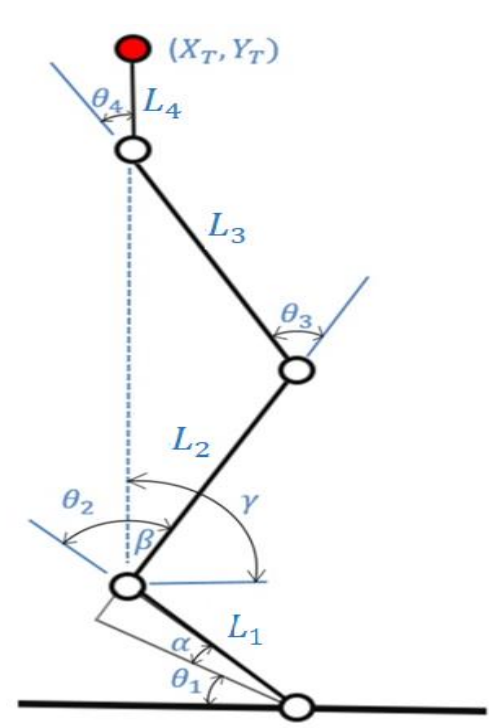

Figure 8. Biomechanical leg model with a fixed toe joint position to perform the first stage of the stance motion.

$$
\begin{aligned}
A & =\left[\begin{array}{ccc}
M L_{1}^{2} & M L_{1} L_{2} C_{2-1} & M L_{1} L_{3} C_{3-1} \\
M L_{1} L_{2} C_{2-1} & M L_{2}^{2} & M L_{2} L_{3} C_{3-2} \\
M L_{1} L_{3} C_{3-1} & M L_{2} L_{3} C_{3-2} & M L_{3}^{2}
\end{array}\right] \\
B & =\left[\begin{array}{ccc}
0 & -M L_{1} L_{2} S_{2-1} & -M L_{1} L_{3} S_{3-1} \\
M L_{1} L_{2} S_{2-1} & 0 & -M L_{2} L_{3} S_{3-2} \\
M L_{1} L_{3} S_{3-1} & M L_{2} L_{3} S_{3-2} & 0
\end{array}\right] \\
G & =\left[\begin{array}{l}
-M g L_{1} C_{1} \\
-M g L_{2} C_{2} \\
-M g L_{3} C_{3}
\end{array}\right],
\end{aligned}
$$

where $S_{n-m}$ and $C_{n-m}$ are defined as $\operatorname{Sin}\left(\theta_{n}-\right.$ $\left.\theta_{m}\right)$ and $\cos \left(\theta_{n}-\theta_{m}\right)$, respectively. By using the toque equation (28) and the angular velocity of each joint $\dot{\theta}_{n}$, the output energy of the joints can be calculated from the following equation:

$W_{n}=\int_{0}^{t}\left|\tau_{n} \cdot \dot{\theta_{n}}\right| d t$

\section{Results and Discussion}

The role of the toe joint comes into place in the biomechanical leg, during the normal gait, at the end of the stance phase and in the first stage of the swing phase. These two stages are addressed by the movements between position " 4 " to position " 5 " and from position " 5 " to position "6" (Fig. 4). The study by Wang et al. (2006) showed the advantages of using the passive toe joint in the biped robot without considering any design factors for the passive toe joint. In Yamamoto et al. 2007; and Zhu et al. 2010, the design factors and the dynamics (i.e. stiffness and damping coefficients) of the passive toe joint were considered for only one case. However, in this study, three different cases were presented, which show the effect of considering different values for the design factors in the passive toe joint in the energy consumption of the biomechanical leg.

Figure 9 compares the joints' angles of the biomechanical leg, during the last stage of the stance motion, for the four toe joint cases. $\theta_{1}, \theta_{2}$, $\theta_{3}$, and $\theta_{4}$ represent the toe, ankle, knee, and hip joints, respectively. Except in Case 4, the case with flat-foot (fixed toe-joint), the start and the end values for all joints are the same. However, the trajectories of these joints values are different in the middle. It is very clear from the figure that the minimum changes in the ankle $\left(\theta_{2}\right)$ and the knee $\left(\theta_{3}\right)$ joints appear in Case 1.

The effect of the presence of the toe joint is apparent in the toque graphs (Fig. 10). $\tau_{1}, \tau_{2}$, and $\tau_{3}$ represent the toe, ankle, and the knee torques, respectively. In Case 1, although torque of the knee joint $\left(\tau_{3}\right)$ starts with a high value compared to the other cases, the torque of the knee joint in Case 1, has a lower value compared to the other cases during the whole motion. Another feature of Case 1 is that the toque of the ankle joint $\left(\tau_{2}\right)$ starts with a very low value and continues to have a minimal toque value during the motion compared to the other three cases.

Figure 11 shows the output energy curves for the joints during the desired motion for the four cases. $E_{1}, E_{2}$, and $E_{3}$ represent the toe, ankle, and the knee energies, respectively. In both Fig. $11\left((a)\right.$, and (b)) the total energy consumption $\left(E_{1}\right.$ and $E_{2}$ ) by the toe and the ankle joints, respectively, during the 1.5 seconds gait for the three designed cases (Case 1, Case 2, and Case 3) are approximately the same. Since there is a proportional relation between the energy and the torque, the results for the energy (Fig. 11(c)) were expected where the energy of the knee joint $\left(E_{3}\right)$, in Case 1 , started from a higher value compared to the other three cases. However, during the rest of the motion, it maintains a minimal value. Case 2 was competing with Case 3 in having minimal energy output for knee joint $\left(E_{3}\right)$ in the early stage of the motion and the final energy outcome of both cases where it was 


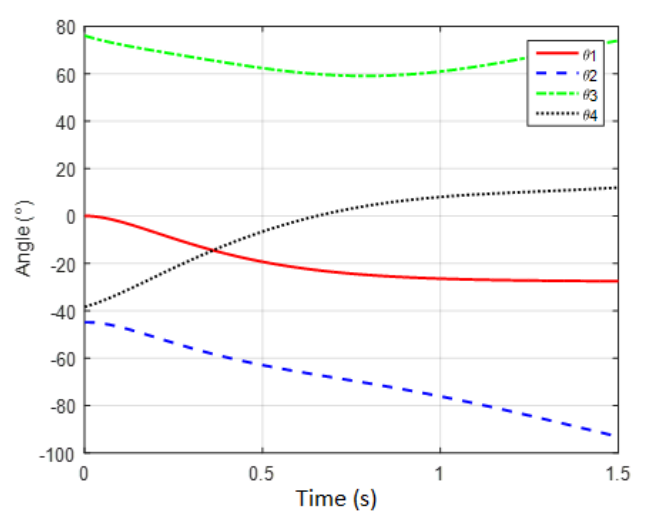

(a) Case 1

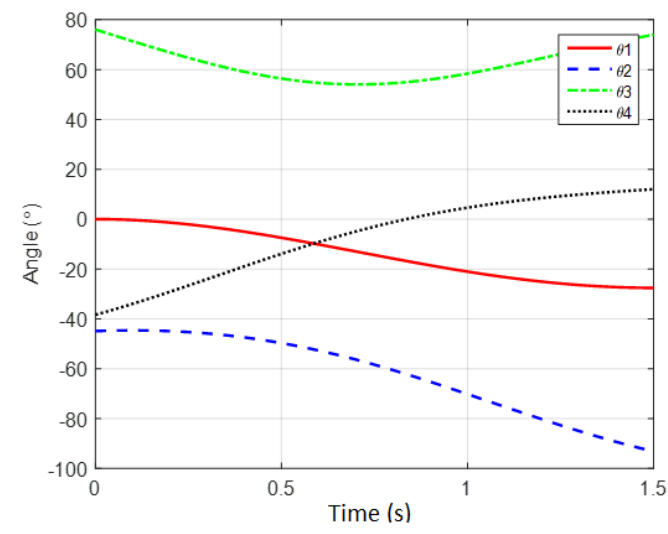

(c) Case 3

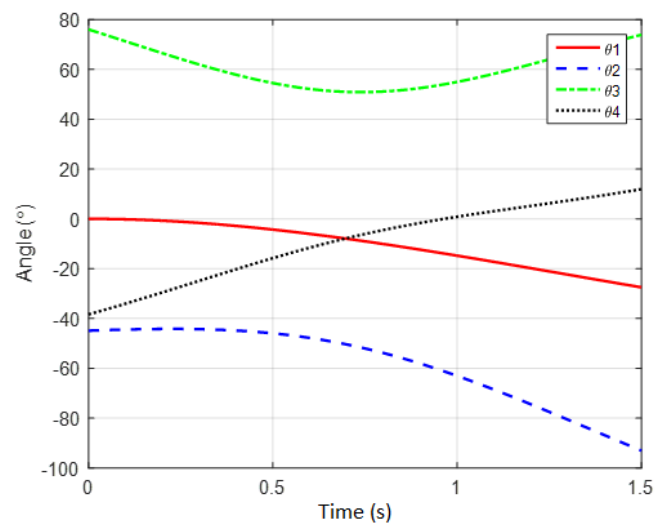

(b) Case 2

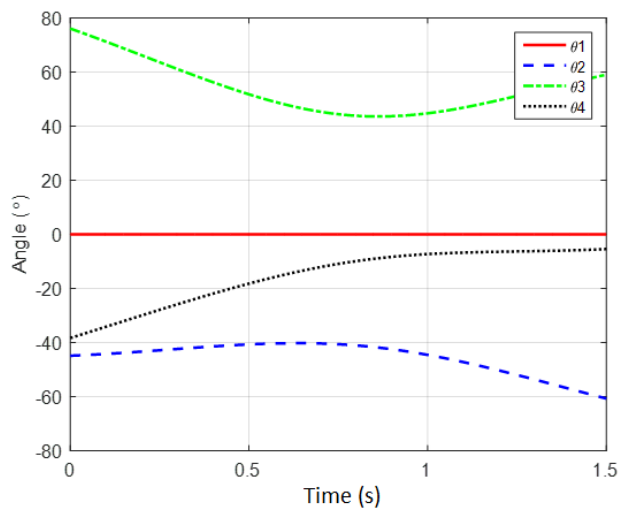

(d) Case 4

Figure 9. The calculated changes in the joints angles $\left(\theta_{2}, \theta_{3}\right.$, and $\left.\theta_{4}\right)$ for a given torso movement, with different trajectories (different cases) of toe joint $\left(\theta_{1}\right)$.

less than the outcome of Case 4 (without the toe joint). In the desired motion, the greatest reduction in energy consumption due to the presence of the toe joint was found in the knee $\left(\theta_{3}\right)$ joint. The greatest reduction was found to be in Case 1 with $k_{1}=125(\mathrm{~N} / \mathrm{m})$, and $c_{1}=50(\mathrm{~N} . \mathrm{s} / \mathrm{m})$. Since these calculations were based on the linear spring and damper coefficients, then, the rotational spring and damper elements of the system can be calculated, using equations (5) and (6). The calculations of Case 1 give $k_{\mathrm{r}}=2.1125$ the rotational stiffness and $c_{\mathrm{r}}=0.845$ (m.N.s/rad) as the damping coefficient for the rotational model. Moreover, the overall output energy of the knee joint for the designed case (with the toe-joint) is less than the output energy of the knee joint in the case without the toe joint.

\section{Conclusion}

This study presented the design procedure for biomechanical legs with a passive toe joint. The design was made based on the motion capability of normal human gait where the joints' ranges of human joints are considered. The design of these biomechanical legs was made from very light materials. The dimensions of the designed leg and the motors of the joints were selected so that the biomechanical leg could mimic the gait of normal human.

A unique design for a toe joint was presented, in detail, to address the concept of the passive joint and to present its role during the human-like motion. The suggested design of the toe joint allowed for different design cases with different design parameters to be examined and tested. The proposed model for the passive toe joint was presented as a massspring damper system and three designed cases were extracted from this model. Another case was introduced for the foot as a flat-foot (fixed toe joint). The results, from the dynamic analysis that compares these four cases, gave a noticeable finding on how the presence of the 


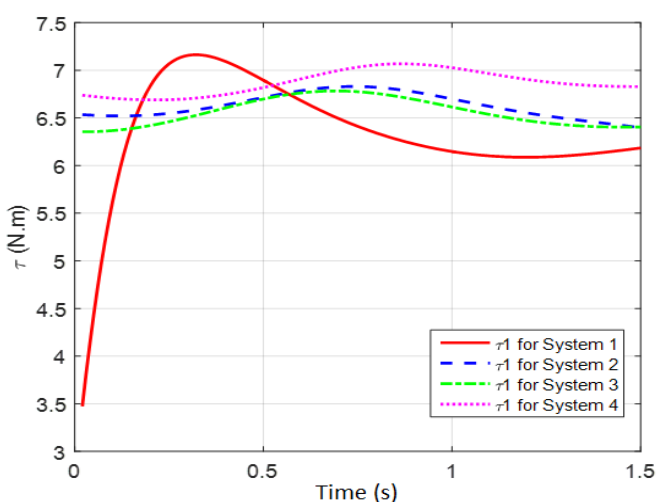

(a)

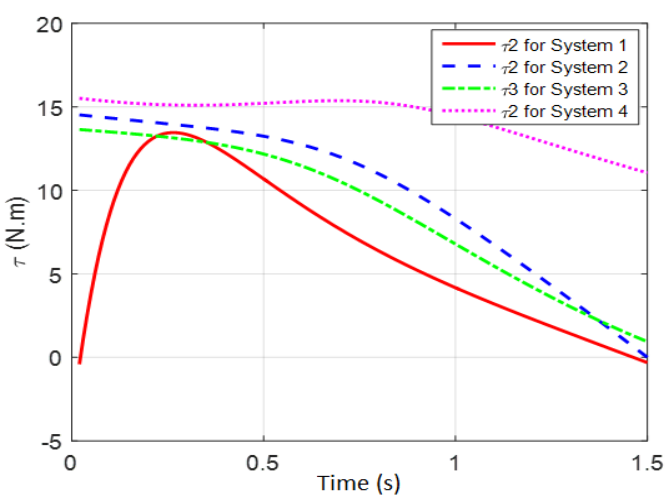

(b)

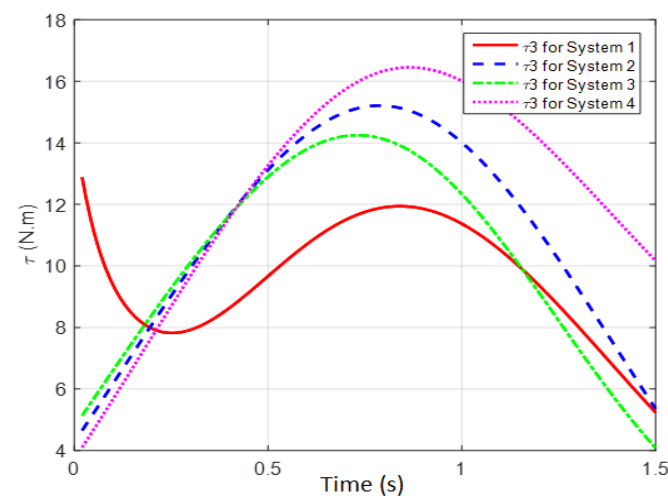

(c)

Figure 10. The calculated torques in the joints: (a) Toe, (b) Ankle, and (c) Knee for a given torso movement, with different trajectories (different cases) of toe joint $\left(\theta_{1}\right)$.

passive top joint can affect the performance of proposed design, the reduction in energy consumption by the actuated joints, due to the presence of the passive toe joint, was proved. The case with the least energy consumption was identified. The particular case will be used when designing the final design for the manufacturing phase. Moreover, the results

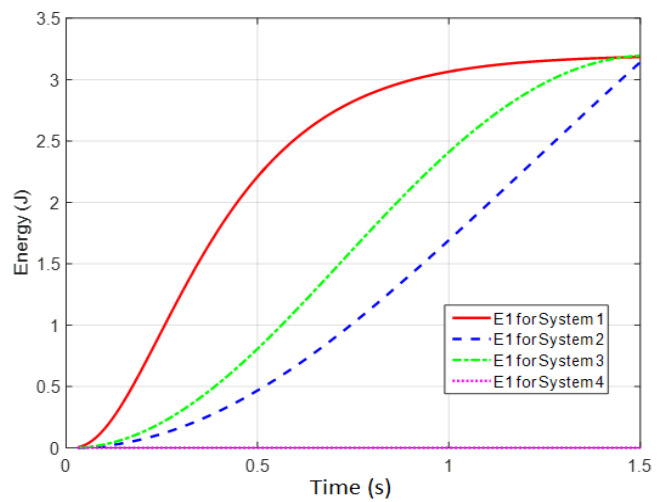

(a)

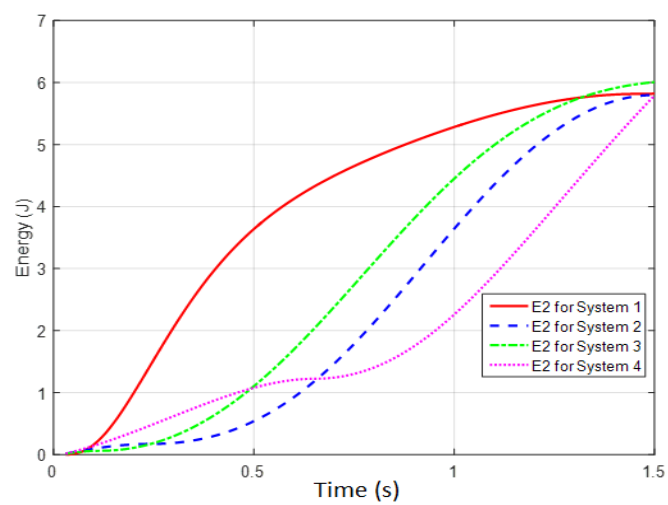

(b)

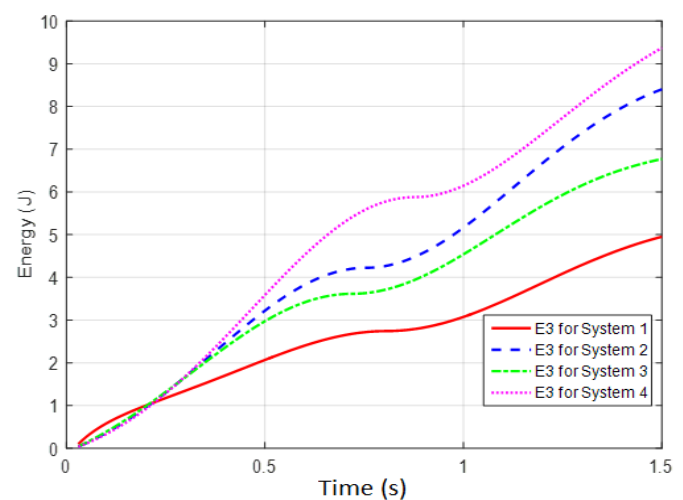

(c)

Figure 11. The calculated energy in the joints (a) Toe, (b) Ankle, and (c) Knee for a given torso movement, with different trajectories (different cases) of toe joint $\left(\theta_{1}\right)$.

from this paper gave an idea about how the driven trajectory of the passive toe joint, by the powered joints, can affect the dynamic motion of a biomechanical leg.

\section{Conflict of Interest}

The authors declare no conflicts of interest. 


\section{Funding}

No funding was received for this research.

\section{References}

Alghooneh M, Wu CQ, Esfandiari M (2016), A passive-based physical bipedal robot with a dynamic and energy efficient gait on the flat ground. IEEE International Conference on Transactions on Mechatronics 21(4): 1977-84.

Allemand Y, Stauffer Y, Clavel R, Brodard R (2009), Design of a new lower extremity orthosis for overground gait training with the walk trainer. IEEE International Conference on Rehabilitation Robotics 550-55.

Au SK, Weber J, Herr H (2007), Biomechanical design of a powered ankle-foot prosthesis. IEEE 10th International Conference on Rehabilitation Robotics 298-303.

Collins SH, Wisse M, Ruina A (2001), A threedimensional passive-dynamic walking robot with two legs and knees. The International Journal of Robotics Research 20(7): 607-15.

Collins S, Ruina A, Tedrake R, Wisse M (2005), Efficient bipedal robots based on passivedynamic walkers. Science 307(5712): 1082-85.

De Leva P (1996), Adjustments to zatsiorskyseluyanov's segment inertia parameters. Journal of Biomechanics 29(9): 1223-30.

Denny J, Elyas M, D'costa SA, D'Souza RD (2016), Humanoid Robots-Past, Present and the Future. European Journal of Advances in Engineering and Technology 3(5): 8-15.

Ezati M, Khadiv M, Moosavian SAA (2014), Dynamics modeling of a biped robot with active toe joints. In Second RSI/ISM International Conference on Robotics and Mechatronics (ICRoM) 107-12.

Grasso R, Zago M, Lacquaniti F (2000), Interactions between posture and locomotion: motor patterns in humans walking with bent posture versus erect posture. Journal of Neurophysiology 83(1): 288300.

Hernández-Santos C, Rodriguez-Leal E, Soto R, Gordillo JL (2012), Kinematics and dynamics of a new 16 DOF humanoid biped robot with active toe joint. International Journal of Advanced Robotic Systems 9(5): 190.

Hwang KS, Lin JL, Li JS (2016), Biped balance control by reinforcement learning. Journal Information Science and Engineering 32(4): 1041-1060.

Iida F, Rummel J, Seyfarth A (2007), Bipedal walking and running with compliant legs.

IEEE International Conference on Robotics and Automation 3970-75.

Iida F, Minekawa Y, Rummel J, Seyfarth A (2009), Toward a human-like biped robot with compliant legs. Robotics and Autonomous Systems 57(2): 139-44.

Inman DJ (2013), Engineering vibration. Pearson 4th Edition.

LaPre AK, Umberger BR, Sup FC (2016), A robotic ankle-foot prosthesis with active alignment. Journal of Medical Devices 10(2): 025001.

McGeer T (1990), Passive dynamic walking. The international Journal of Robotics Research 9(2): 62-82.

Nishiwaki K, Kagami S, Kuniyoshi Y, Inaba M, Inoue $H$ (2002), Toe joints that enhance bipedal and fullbody motion of humanoid robots. In IEEE/ ICRA'02 International Conference on Robotics and Automation (3): 3105-10.

Otani T, Hashimoto K, Isomichi T, Sakaguchi M, Kawakami Y, Lim HO, Takanishi A (2016), Joint mechanism that mimics elastic characteristics in human running. Machines 4(1): 5 .

Piazza C, Della Santina C, Gasparri GM, Catalano MG, Grioli G, Garabini M, Bicchi A (2016), Toward an adaptive foot for natural walking. In IEEE-RAS 16th International Conference on Humanoid Robots (Humanoids) 1204-1210.

Riemer R, Shapiro A (2011), Biomechanical energy harvesting from human motion: theory, state of the art, design guidelines, and future directions. Journal of Neuroengineering and Rehabilitation 8(1): 22.

Robotis (2017), DYNAMIXEL PRO [Online]. Available: Http:/ / en.robotis.com / index / index.php. [Accessed: 10- Oct- 2017].

Sellauoti R, Stasse O, Kajita S, Yokoi K, Kheddar A (2006), Faster and smoother walking of humanoid HRP-2 with passive toe-joints. In IEEE/RSJ International Conference on Intelligent Robots and Systems 4909-14.

Wang L, Yu Z, Meng Q, Zhang Z (2006), Influence analysis of toe-joint on biped gaits. 
In 2006 International Conference on Mechatronics and Automation 1631-35.

Wisse M, Van Frankenhuyzen J (2006), Design and construction of MIKE; a 2-D autonomous biped based on passive dynamic walking. In Adaptive Motion of Animals and Machines, Springer Tokyo, 14354.

Yabunaka T, Kawaguchi N, Shinoda N, Nakanishi A, Kobayashi N, Yano KI (2013), Development of an improved lower limb orthosis for a motion-assist robot for the lower limb. In 16th International Conference on Advanced Robotics (ICAR) 1-6.

Yamamoto K, Sugihara T, Nakamura Y (2007), Toe joint mechanism ssing parallel four-bar linkage enabling humanlike multiple support at toe pad and toe tip. In 2007 7th IEEE-RAS International Conference on Humanoid Robots 410-15.
Yi SJ, Zhang BT, Hong D, Lee DD (2016), Whole-body balancing walk controller for position controlled humanoid robots. International Journal of Humanoid Robotics 13(01): 1650011.

Zhu J, Wang Q, Wang L (2010), PANTOE 1: Biomechanical design of powered ankle-foot prosthesis with compliant joints and segmented foot. In 2010 IEEE/ASME International Conference on Advanced Intelligent Mechatronics 31-36. 